\title{
Origins of anomalous electronic structures of epitaxial graphene on silicon carbide
}

\author{
Seungchul Kim, ${ }^{1}$ Jisoon Ihm, ${ }^{1}$ Hyoung Joon Choi, ${ }^{2}$ and Young-Woo Son ${ }^{3}, *$ \\ ${ }^{1}$ Department of Physics and Astronomy, Seoul National University, Seoul 151-747, Korea \\ ${ }^{2}$ Department of Physics and IPAP, Yonsei University, Seoul 120-749, Korea \\ ${ }^{3}$ Department of Physics, Konkuk University, Seoul 143-701, Korea
}

(Dated: August 21, 2021)

\begin{abstract}
On the basis of first-principles calculations, we report that a novel interfacial atomic structure occurs between graphene and the surface of silicon carbide, destroying the Dirac point of graphene and opening a substantial energy gap there. In the calculated atomic structures, a quasi-periodic $6 \times 6$ domain pattern emerges out of a larger commensurate $6 \sqrt{3} \times 6 \sqrt{3} R 30^{\circ}$ periodic interfacial reconstruction, resolving a long standing experimental controversy on the periodicity of the interfacial superstructures. Our theoretical energy spectrum shows a gap and midgap states at the Dirac point of graphene, which are in excellent agreement with the recently-observed anomalous angle-resolved photoemission spectra. Beyond solving unexplained issues in epitaxial graphene, our atomistic study may provide a way to engineer the energy gaps of graphene on substrates.

PACS numbers: 73.20.-r,81.05.Uw,68.35-p,71.20-b
\end{abstract}

Graphene, a carbon allotrope, is a two-dimensional hexagonal network of carbon atoms which is formed by making strong triangular $\sigma$-bonds of the $s p^{2}$ hybridized orbitals [1, 2]. The $\pi$-orbitals orthogonal to the hexagonal plane of graphene are responsible for its characteristic electronic properties, i.e., a relativistic dispersion relation near the Fermi level described by the massless free particle Dirac equation [1, 2, 3, 4]. Following the report of successful fabrication of mechanically exfoliated graphene on the insulating $\mathrm{SiO}_{2}$ surface [3, 4], tremendous efforts have been devoted to measure and exploit the novel physical properties of graphene [1, 2].

On the other hand, it has been known for the last three decades that, when a wide band gap semiconductor silicon carbide ( $\mathrm{SiC})$ is heated up to $1300^{\circ} \mathrm{C}$, the monocrystalline graphite forms on the $\mathrm{SiC}(0001)$ face $[5,6,6,8,9,10,11,12$. Now, by fine tuning the growth parameters, a single layer of graphene can be grown successfully on $\mathrm{SiC}[13,14]$. These researches have stimulated interests in resolving fundamental material properties [5, 6] as well as applying the techniques to nanoelectronics 13, 14], with the merits of precise control of the number of layers of graphene [15, 16, 17] and a possible large scale production [14].

Epitaxial graphene has demonstrated different physical properties compared to exfoliated graphene, exhibiting many controversial experimental observations [12, 13, 14, 15, 16, 17, 18, 19, 20, 21]. For example, scanning tunnelling microscopy (STM) images [12, 13, 14, 18, 19] show a $6 \times 6$ hexagonal superstructure with respect to the surface unitcell of $4 \mathrm{H}-\mathrm{SiC}(0001)$ while low-energy electron diffraction (LEED) patterns indicate a larger scale reconstruction with a $6 \sqrt{3} \times 6 \sqrt{3} R 30^{\circ}$ periodicity $[5,66,7,6$, 9, 10, 11, 12, 13, 14, 18, 20]. Moreover, the energy spectrum from STM [19] and the angle-resolved photoemission spectroscopy (ARPES) measurements [21] show the energy gap which still defy precise interpretations 22 ].
Since the potential profiles induced by interfacial atoms will play a decisive role in the physical properties of graphene grown on $\operatorname{SiC}(0001)$ 1, 2, 21, 22, 23], it is indeed required to know the precise atomic geometries and the corresponding electronic structures of the system. However, in spite of many experimental observations, the atomic structures of the graphene (graphite)-SiC(0001) interfaces have not been uncovered yet except that they have a large scale reconstruction with the $6 \sqrt{3} \times 6 \sqrt{3} R 30^{\circ}$ periodicity [5, 6, 7, 8, 9, 10, 11, 12, 13, 14, 18, 20].

In this Letter, we identify atomic and electronic structures of epitaxial graphene on $4 \mathrm{H}-\mathrm{SiC}(0001)$ by largescale first-principles calculations. In the relaxed atomic structures of the $6 \sqrt{3} \times 6 \sqrt{3} R 30^{\circ}$ periodicity with a single layer graphene, a quasi-periodic $6 \times 6$ domain pattern appears, resolving the aforementioned disagreement between LEED patterns and the STM images. The obtained novel domain pattern originates from interplay between strong bonding and lattice mismatch at the graphene-SiC(0001) interface. With inclusion of another layer of graphene, the calculated electronic structures show a gap opening and midgap states at the Dirac point of graphene, originating from sublattice symmetry breaking interactions between graphene and the interfacial superstructure in the system. Simulated STM images and simulated ARPES spectra for the obtained atomic structures show excellent agreements with several experimental data [12, 13, 14, 18, 19, 20, 21]. Our study resolved the fundamental issues regarding the role of interfaces and substrates in altering physical properties of graphene, and thus provides a way to control the energy gaps of graphene on substrates.

We study the atomic and electronic structures of graphene and interfacial carbons on $4 \mathrm{H}-\mathrm{SiC}(0001)$ based on $a b$ initio pseudopotential density functional methods [24] within the local density approximation [25] which are known to describe the structural and electronic prop- 


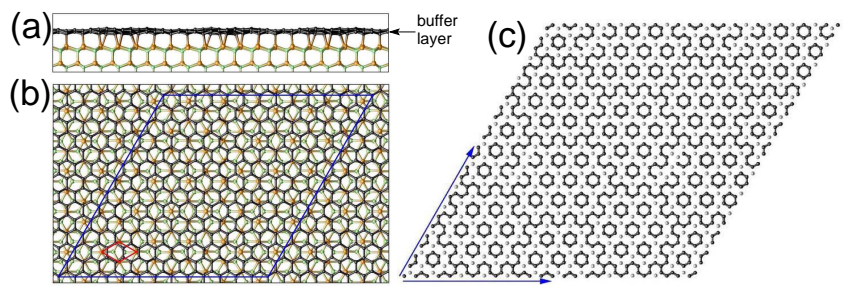

FIG. 1: (color online) (a) Side and (b) top views of the atomic structure of the buffer layer with the $6 \sqrt{3} \times 6 \sqrt{3} R 30^{\circ}$ periodicity. The $6 \sqrt{3} \times 6 \sqrt{3} R 30^{\circ}$ supercell is denoted with blue lines and the $1 \times 1$ surface unit cell of $4 \mathrm{H}-\mathrm{SiC}(0001)$ with red. The carbon atoms in the buffer layer are denoted with black spheres and the silicon and the carbon atoms in the $4 \mathrm{H}$ $\mathrm{SiC}$ with orange and green spheres, respectively. (c) Bonding characteristics of carbon atoms in the buffer layer. Carbon atoms with (without) $\sigma$-bonding to surface silicon atoms are represented with grey (black) dots. The $\pi$-bonds, represented by black lines, form a super-hexagonal pattern. Four times the $6 \sqrt{3} \times 6 \sqrt{3} R 30^{\circ}$ periodicity is drawn in order to display the super-hexagons clearly. The blue arrows are the unit vectors of the $6 \sqrt{3} \times 6 \sqrt{3} R 30^{\circ}$ supercell.

erties of graphite quite well [26, 27]. The $4 \mathrm{H}-\mathrm{SiC}(0001)$ substrate is modelled with four alternating silicon and carbon atomic layers, and one or two graphene layers are placed on top of the $\mathrm{SiC}$ substrate. The atoms belonging to the bottom layer of the slab are passivated by hydrogen. To incorporate a large number of atoms in the system, we expand the wave function with localized basis sets [28]. The basis sets and the pseudopotentials are thoroughly tested to reproduce the atomic and electronic structures of $\mathrm{SiC}$, graphene and the $\sqrt{3} \times \sqrt{3} R 30^{\circ}$ model for epitaxial graphene studied in previous literatures [26, 27] respectivley. Based on the LEED measurements [5, 6, 7, 8, 9, 10, 11, 12, 13, 14, 18, 20], the $6 \sqrt{3} \times 6 \sqrt{3} R 30^{\circ}$ periodicity is imposed to the $\mathrm{SiC}(0001)$ surface, which is equivalent to $13 \times 13$ times a graphene unit cell [Fig 1(a), (b)]. The atomic positions are determined by minimizing the total energy until the forces on each atom are less than $0.06 \mathrm{eV} / \AA$ while atoms belonging to the last two silicon and carbon layers are fixed to the bulk atomic structure of $4 \mathrm{H}-\mathrm{SiC}$. To simulate ARPES spectra, wavefunctions of the $6 \sqrt{3} \times 6 \sqrt{3} R 30^{\circ}$ supercell obtained with a few k-points are Fourier-transformed to the surface reciprocal space of graphene unit cell with dense k-points and then integrated along the surface normal direction with attenuation corresponding to a photon mean free path of $5 \AA$. The calculated spectra are broadened by $30 \mathrm{meV}$ in energy.

The obtained atomic structure, in the case of one layer of graphene on the $\mathrm{SiC}$ surface, displays a novel pattern of covalent bonding between carbon atoms in graphene and silicon atoms on the (0001) face [Fig. 1(c)]. We find that, due to the interplay of lattice mismatch and strong C-Si bonds, the $6 \sqrt{3} \times 6 \sqrt{3} R 30^{\circ}$ supercell is split into lattice matched regions, where carbon atoms are covalently

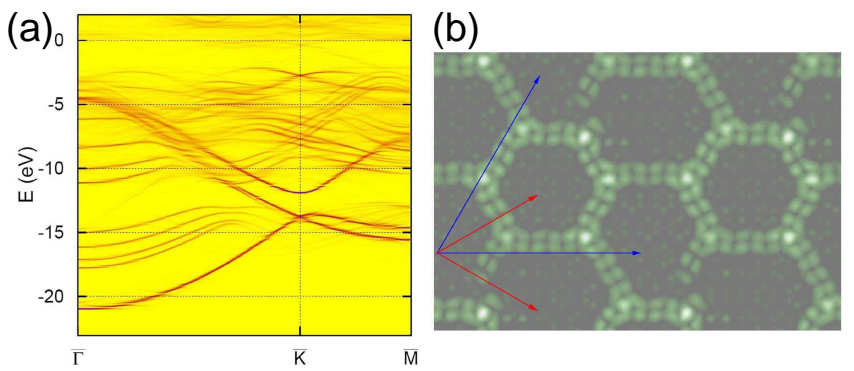

FIG. 2: (color online) (a) Simulated spectrum for ARPES of the buffer layer on top of $4 \mathrm{H}-\mathrm{SiC}(0001)$ in the graphene Brillouin zone. (b) Simulated STM image of the structure. Wavefunctions of which energies lie between the Fermi level $\left(E_{F}\right)$ and $0.2 \mathrm{eV}$ above $E_{F}$ are integrated and the image is taken in a plane located at $3 \AA$ above the buffer layer. Bright (dark) regions correspond to a high (low) current in constantheight mode for STM. The $6 \sqrt{3} \times 6 \sqrt{3} R 30^{\circ}$ periodic lattice vectors are drawn in blue while $6 \times 6$ periodic ones in red.

bonded to surface silicon atoms with $\sqrt{3} \times \sqrt{3} R 30^{\circ}$ periodicity with respect to the surface unitcell of $\mathrm{SiC}(0001)$. Outside the lattice matched regions, there are boundaries consisting of lines of carbon atoms (connected or disconnected with each other) without covalent bonding to surface silicon atoms. Our calculations show that the covalent bonding in the regions cannot be sustained over three or four units of the reconstruction and that the carbon atoms with (without) covalent $\sigma$-bonding to silicon atoms move toward (away from) the $\mathrm{SiC}$ substrate. The small regions of covalent bondings follow the lattice symmetry of graphene so that all the lattice matched regions are shown to have large hexagonal shapes approximately [Fig. 1]. We find that the novel hexagonal pattern appearing in the present simulation is quite robust and does not depend on the details of calculations.

Our atomic structure for a single layer graphene on the SiC substrate [Fig. 1] is consistent with several experimental observations for the initial stage of graphene formation $7,8,19,10,11,12,13,14,18,19,20$, in which the observed structure is called a carbon nanomesh 12 . or a buffer (or dead) layer 16, 21, 26, 27]. We will call it a buffer layer hereafter. First of all, our model for the buffer layer has the $6 \sqrt{3} \times 6 \sqrt{3} R 30^{\circ}$ reconstruction satisfying all the LEED measurements [5, 6, 7, 8, 9, 10, 11, 12, 13, 14, 18, 20. Second, our simulated spectrum for the ARPES shows that $\sigma$-bands in the buffer layer appear clearly while the linear $\pi$-bands around the Fermi energy are absent [Fig. 2(a)], compatible with the recent ARPES measurement 20]. Instead, there are several flat bands above and below the Fermi energy originating from $\pi$-orbitals of carbon atoms on the super-hexagonal boundaries. In our atomic structure [Fig. 1], the covalent bonding between graphene and the $\mathrm{SiC}$ surface breaks the hexagonal network of $\pi$-orbitals but preserves $\sigma$-bonds of $s p^{2}$ hybridization. Hence, the resulting band structure in 
the graphene Brillouin zone [Fig. 2(a)] shows no relativistic dispersion relation near the Fermi level of the system. Third, our simulated STM image shows an approximate $6 \times 6$ periodicity with respect to the surface unit cell of $\mathrm{SiC}(0001)$ [Fig. 2(b)]. This also bears a striking similarity to the observed STM images [12, 13, 14, 18, 19, 29], although the $6 \sqrt{3} \times 6 \sqrt{3} R 30^{\circ}$ periodicity is imposed on our atomic structure. The bright regions in the simulated STM image [Fig. 2(b)] originate from the (broken) chains of $\pi$-orbitals with approximate super-hexagonal shapes in large scale and the dark regions correspond to domains of carbon atoms having strong $\sigma$-bonds to the surface silicon atoms. Our atomic model for the buffer layer has a complete coverage of carbon atoms on the $\mathrm{SiC}(0001)$ surface without any silion atoms on it. The model is compatible with not only the aforementioned spectroscopic measurement [20] but also recent STM studies on the adsorption of metallic clusters [30] and fullerenes 31] on the buffer layer indicating clean carbon surfaces.

Next, we consider another graphene layer on top of the reconstructed buffer layer on $4 \mathrm{H}-\mathrm{SiC}(0001)$ surface [Fig. 3(a)]. The calculated atomic structure shows an almost free standing graphene with corrugations following the atomic structures underneath it [Fig. 3(a)]. The corrugation height is $\pm 0.17 \AA$, with the mean distance of 3.35 $\AA$ from the buffer layer. The simulated STM image [Fig. $3(\mathrm{~b})$ ] shows that all hexagonal networks of carbon atoms are clearly visible and approximate $6 \times 6$ periodic large hexagonal shapes are superimposed on it. This matches very well with the existing experimental data [14, 18, 19], although the $6 \sqrt{3} \times 6 \sqrt{3} R 30^{\circ}$ periodicity is imposed on our atomic structure. In our calculations, the apparent $6 \times 6$ periodicity originates both from the atomic corrugations due to the underlying buffer layer and from the weak electronic interaction between graphene and the buffer layer. Thus, our results resolve the disagreement between the $6 \times 6$ periodicity from STM measurements and the $6 \sqrt{3} \times 6 \sqrt{3} R 30^{\circ}$ from the LEED measurements.

We find that the simulated ARPES spectrum of graphene on top of the buffer layer show the characteristic $\pi$-bands of graphene as well as $\sigma$-bands [Fig. 3(c)]. The crossing point of two linear $\pi$-bands of graphene (called as the Dirac point [1, 2]) is located slightly below the Fermi energy of the system. However, if looked closely, there is a gap opening at the Dirac point. We shall defer the discussion of the gap later. From Mullikan population analysis [24], graphene is found to be electron-doped with a density, $n \simeq 8.7 \times 10^{12} / \mathrm{cm}^{2}$, consistent with experimental observations [15, 16, 17, 20, 21]. It is also noticeable that the $\sigma$-bands of graphene are rigidly shifted up in energy compared with those of the buffer layer. This shift is observed in the recent ARPES measurement [20] and the size of the shift $(1.2 \mathrm{eV})$ is consistent with their observations 20]. The energy shift of the $\sigma$-bands of graphene arises from a potential gradient due to a polar nature of the $\mathrm{SiC}$ surface.

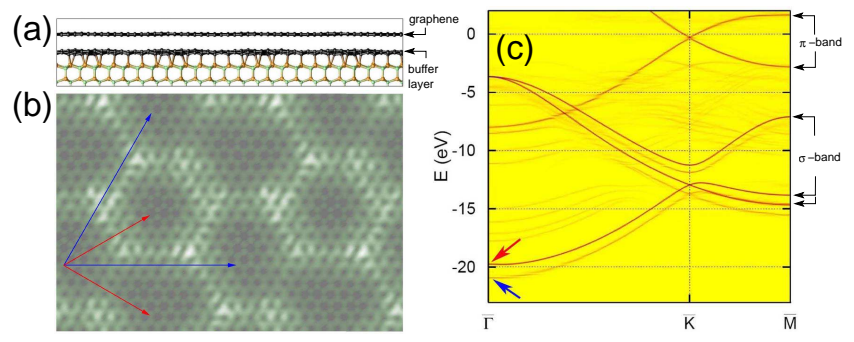

FIG. 3: (color online) (a) Side view of the atomic structure of graphene on the buffer layer with the $6 \sqrt{3} \times 6 \sqrt{3} R 30^{\circ}$ periodicity. (b) Simulated STM image of graphene shown in (a). The unit vectors for the $6 \sqrt{3} \times 6 \sqrt{3} R 30^{\circ}$ periodicity are drawn in blue arrows and those for $6 \times 6$ in red. (c) Simulated ARPES spectrum for graphene shown in (a). The bottom of $\sigma$-bands in graphene is shown to be shifted up in energy (pointed by red arrow) compared with that in the buffer layer (blue arrow). The simulated STM image are obtained by the same method as in Fig. 2.

The electronic structure near the Dirac point shows a gap of $200 \mathrm{meV}$ with the centre of the gap located at 320 meV below the Fermi energy [Fig. 4(a)]. It also shows midgap states inside the gap. The spectrum exhibiting the gap and midgap states is very similar with the recent ARPES observations [21]. The gap at the Dirac point in our calculation originates mainly from the interlayer coupling between graphene and the buffer layer which breaks a sublattice symmtery in graphene [2, 21, 22, 23]. Considering the valley and pseudo-spin symmetries exhibited in the electronic structure of graphene [1, 2], there are two possible ways of inducing a gap at Dirac points in a single layer of graphene. One is the mixing of electronic states with different pseudo-spins in the same valley and the other is the mixing of states belong to different valleys [2, 21, 22, 23]. Due to the presence of the buffer layer, there exists a substantially different interaction at atomic sites belonging to each sublattice of graphene [Fig. 4(b)], breaking the sublattice symmetry. The resulting wavefunctions near the Dirac point reflect such a broken symmetry so that the weight of the wavefunction on one sublattice is predominant over the other [Fig. 4(c)]. The intervalley mixing is found to have a negligible contribution to the gap. In case that the substrate is intentionally removed from the system in the calculation, the remaining graphene with present corrugations does not show any gap at the Dirac point. The midgap states originate from the interlayer coupling (the hopping energy of $0.2 \sim 0.3 \mathrm{eV}$ between $\pi$-orbitals [2]) between the $\pi$-states in graphene and the localized $\pi$-states on the boundaries between super-hexagons in the buffer layer [Fig. 4(d)]. The corresponding wavefunctions spatially spread out into graphene (the topmost surface of the system) [Fig. 4(d)] and thus can be detected by surface sensitive measurements such as ARPES. It explains the reason why the energy distribution curve shown in 

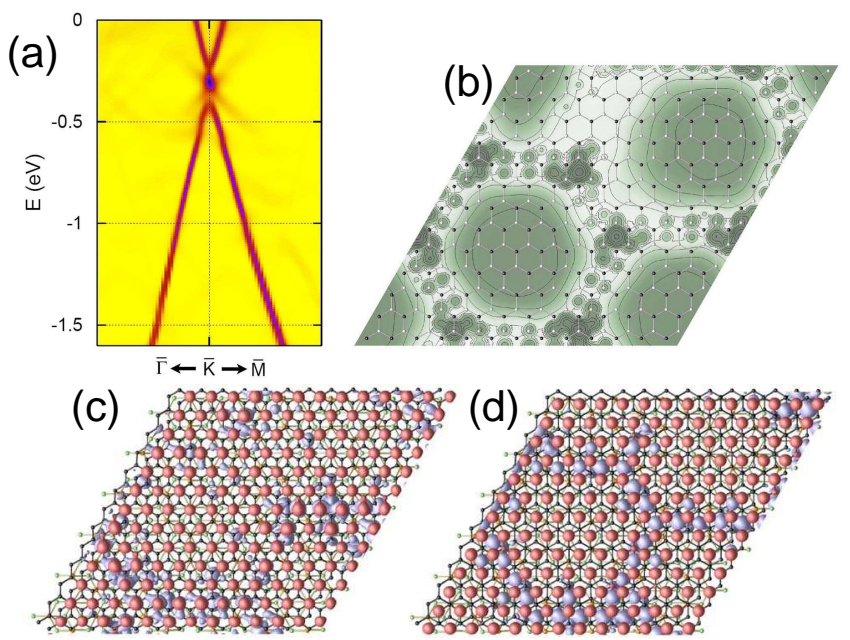

FIG. 4: (color online) (a) The magnified view of the ARPES spectrum near the Dirac point. (b) Contour plot (contour spacing $=0.1 \mathrm{eV}$ ) for the potential generated by the buffer layer and $\mathrm{SiC}$ substrate only, drawn on a plane located at $3.35 \AA$ above the buffer layer. The bright (dark) color corresponds to high (low) potential. The hexagonal network (grey lines) for graphene is drawn to guide the eyes. The dark and bright dots represent two sublattices having $\sim 140 \mathrm{meV}$ averaged potential difference, respectively. (c) The squared amplitude of wavefunctions (isosurface of $3.0 \times 10^{-4} / \AA^{3}$ ) of which energy is located at the upper apex of the energy bands at $\mathrm{K}$ (shown in (a)). The amplitude of the wavefunction at graphene is denoted in red while the one at the buffer layer in light blue. (d) The squared amplitude of the wavefuntions whose energies are inside the energy gap at Dirac point (the averaged midgap state). The isosurface value is $3.0 \times 10^{-4} / \AA^{3}$. The red and blue colours for the amplitude follow the same scheme in (c).

the recent ARPES measurement 21] has anomalous nonvanishing weights inside the gap at the Dirac point.

In summary, we have demonstrated that the interface between the $\mathrm{SiC}$ (0001) surface and graphene show the novel large scale atomic reconstruction and the fundamental electronic property of epitaxial graphene is altered due to the interface. The present atomistic study on the atomic and electronic structures of epitaxial graphene will play a crucial role not only in designing electronic circuits [14 but also in explaining many other puzzling observations such as the absence of the quantum Hall effect and the weak Shubnikov-de Hass oscillations in the high mobility sample [14, 32].

Y.-W. S. thanks H.-D. Kim and W. Chen for discussions. S. K and J. I acknowledge the support of the SRC program (Center for Nanotubes and Nanostructured Composites) of MOST/KOSEF. H.J.C. acknowledges support from the KRF (KRF-2007-314-C00075) and from the KOSEF Grant No. R01-2007-000-20922-
0. Y.-W. S. was supported by the KOSEF grant funded by the Korea government (MOST) No. R01-2007-00010654-0. Computational resources have been provided by KISTI Supercomputing Center (KSC-2007-S00-1011).

* To whom correspondence should be addressed. E-mail: youngwoo@konkuk.ac.kr

[1] A. K. Geim and K. S. Novoselov, Nature Mat. 6, 183 (2007).

[2] A. H. Castro Neto, F. Guinea, N. M. R. Peres, K. S. Novoselov and A. K. Geim, arXiv:0709.1163.

[3] K. S. Novoselov et al., Nature 438, 197 (2005).

[4] Y. Zhang, Y.-W. Tan, H. L. Stormer and P. Kim, Nature 438, 201 (2005).

[5] U. Starke, Phys. Stat. Sol. (b) 202, 475 (1997).

[6] T. Seyller, Appl. Phys. A 85, 371 (2006).

[7] A. J. van Bommel, J. E. Crombeen and A. van Tooren, Surf. Sci. 48, 463 (1975).

[8] M.-H. Tsai, C. S. Chang, J. D. Dow and I. S. T. Tsong, Phys. Rev. B 45, 1327 (1992).

[9] F. Owman and P. Mårtensson, Surf. Sci. 369, 126 (1996).

[10] I. Forbeaux, J. M. Themlin and J. M. Deveber, Phys. Rev. B 58, 16396 (1998).

[11] A. Charrier et al., J. Appl. Phys. 92, 2479 (2002).

[12] W. Chen et al., Surf. Sci. 596, 176 (2005).

[13] C. Berger et al., Science 312, 1191 (2006).

[14] W. A. de Heer et al., Solid State Commun. 143, 92 (2007).

[15] A. Bostwick, T. Ohta, T. Seyller, K. Horn and E. Rotenberg, Nat. Phys. 3, 36 (2007).

[16] A. Bostwick et al., New J. Phys. 9, 385 (2007).

[17] T. Ohta et al., Phys. Rev. Lett. 98, 206802 (2007).

[18] P. Mallet et al., Phys. Rev. B 76, 041403(R) (2007).

[19] V. W. Brar et al., Appl. Phys. Lett. 91, 122102 (2007).

[20] K. V. Emtsev et al., Mater. Sci. Forum 556, 525 (2007).

[21] S. Y. Zhou et al., Nature Mat. 6, 770 (2007).

[22] K. Novoselov, Nature Mat. 6, 720 (2007).

[23] G. Giovannetti, P. A. Khomyakov, G. Brocks, P. J. Kelly and J. van den Brink, Phys. Rev. B 76, 073103 (2007).

[24] J. M. Soler et al., J. Phys. Condens. Matter 14, 2745 (2002).

[25] D. M. Ceperley and B. J. Alder, Phys. Rev. Lett. 45, 566 (1980).

[26] A. Mattausch and O. Pankratov, Phys. Rev. Lett. 99, 076802 (2007).

[27] F. Varchon et al., Phys. Rev. Lett. 99, 126805 (2007).

[28] We have used a single- $\zeta$ and a single- $\zeta$ plus polarization basis set for hydrogen and silicon atom respectively. For carbon atom, a mixed basis set with a single and double- $\zeta$ for $s$ - and $p$-orbitals respectively, has been used.

[29] F. Varchon, P. Mallet, J.-Y. Veuillen and L. Magaud, arXiv.org:0712.3394.

[30] W. Chen, K. P. Loh, H. Xu and A. T. S. Wee, Langmuir 20, 10779 (2004).

[31] W. Chen et al, J. Phys. Chem. B 110, 21873 (2006).

[32] P. Darancet, N. Wipf and D. Mayou, arXiv:0711.0940. 